\title{
Outcome after stenting and débridement for spontaneous esophageal
}

\section{rupture}

Tobias Hauge $^{\mathrm{a}}$, Ole Christian Kleven ${ }^{\mathrm{b}}$, Egil Johnson ${ }^{\mathrm{c}, \mathrm{d}}$, Bjørn Hofstad ${ }^{\mathrm{e}}$, Hans-Olaf Johannessen ${ }^{c}$

a Department of Surgery, Drammen Hospital, Vestre Viken HF, Drammen; ${ }^{b}$ Department of Surgery, Lillehammer Hospital, Sykehuset Innlandet, Lillehammer, Norway;

cDepartment of Pediatric and Gastrointestinal Surgery, Oslo University Hospital, Ullevål, Oslo, Norway; 'institute of Clinical Medicine, University of Oslo, Oslo,

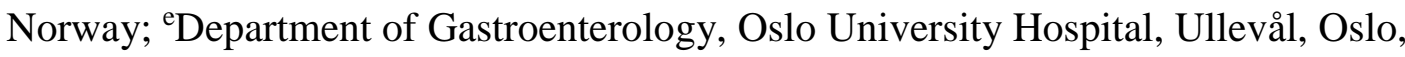
Norway

Tobias Hauge

Department of Surgery, Drammen Hospital, Vestre Viken HF, Drammen Postboks 800, 3004 Drammen, Norway, Phone: 47409035, E-mail:

tobiaha@gmail.com

Social media: Facebook

Ole Christian Kleven

Department of Surgery, Lillehammer Hospital, Sykehuset Innlandet, Lillehammer, Norway

Anders Sandvigs gate 17, 2609 Lillehammer, Phone:0047 41640869

E-mail:oleckl@yahoo.no

Social media: Twitter @OCKleven

Corresponding author:

Egil Johnson

Department of Pediatric and Gastrointestinal Surgery; Oslo University Hospital, Ullevål, Norway, P. O. Box 4956 Nydalen, 0424 Oslo, Norway, Phone: 0047

47416334

E-mail: egil.johnson@ous-hf.no

Social media: Linkedin

Bjørn Hofstad

Department of Gastroenterology, Oslo University Hospital, Ullevål, Oslo, Norway P. O. Box 4956 Nydalen, 0424 Oslo, Norway,

Phone: 0047 99715960, E-mail: uxbjho@ous-hf.no

Hans-Olaf Johannessen

Department of Pediatric and Gastrointestinal Surgery; Oslo University Hospital, Ullevål, Norway

P. O. Box 4956 Nydalen, 0424 Oslo, Norway, Phone: 004799700198

E-mail: hans-olaf.johannessen@ous-hf.no

Social media: Linkedin 


\title{
Outcome after stenting and débridement for spontaneous esophageal rupture
}

\author{
Abstract \\ Objectives: Surgical repair has been the most common treatment of esophageal effort \\ rupture (Boerhaave syndrome). Stent-induced sealing of the perforation has increasingly \\ been used with promising results. We present our eight years' experience with stent- \\ based and organ-preserving treatment.
}

Materials and methods: Medical records of 15 consecutive patients with Boerhaave syndrome from February 2007 to May 2015 were retrospectively registered in a database. Treatment was sealing of the perforation by stenting, chest tube drainage and débridement of the contaminated thorax. Nine out of 10 patients after median 25 months responded to questions on fatigue and Ogilvie's dysphagia score.

Results: Fifteen patients, aged median 67.5 years (range $39-88$ ), had a primary hospital stay of 20 days (range 1 - 80 days). Overall in-hospital mortality was $13 \%$. Observation time was 44 months (range $0-87$ ) and 10 patients were alive of August 2017. Ten patients (67\%) needed surgical chest débridement. Seven patients (47\%) were restented for leakage $(n=5)$, migration and for stent removal. Eleven patients $(73 \%)$ had complications, which included pleural empyema $(n=4)$, fatal aortic bleeding, lung arterial bleeding, lung embolism, drain-induced lung laceration and respiratory failure. Dysphagia score was low (median 0.5) meaning that they were able to feed themselves. Total fatigue score (mean 14.6) was slightly increased $(\mathrm{p}=0.05)$ compared with a reference population.

Conclusion: The mortality rate after initial stenting of effort rupture seems to be comparable to standard surgical repair. Most patients required further intervention, either by restenting and/or surgical débridement. The functional result in these patients was satisfactorily.

Keywords: Boerhaave syndrome, stenting, drainage, débridement, dysphagia, fatigue 


\section{Introduction}

Spontaneous postemetic rupture of the esophagus (Boerhaave syndrome) is a devastating event with high morbidity and mortality, mainly because of diagnostic delay and irreversible gastric contamination of the mediastinum and pleural cavities. Surgery has been the main-stay of treatment [1], including primary repair, resection or exclusion of the perforation combined with drainage and débridement of the contaminated chest. During the past 15 years, sealing of the perforation by placement of a self expanding metal stent (SEMS) has increasingly been used $[2,3]$ with promising results. The main goals of stent-based treatment are to seal and close the perforation effectively, to drain all fluid collections in the pleura and the mediastinum, primarily percutaneously, and perform surgical débridement in selected patients. In the situation of a clearly contaminated and turbid pleural effusion that may contain food remnants, surgical débridement of the chest is mandatory in order to avoid a potentially fatal outcome.

The aim of this study was to report the outcome of treatment of 15 consecutive patients with Borehaave syndrome at Oslo University Hospital from 2007-2015. This included the majority of patients with spontaneous esophageal rupture in the health region of south-east Norway. The initial treatment was based on sealing of the rupture by stenting, transthoracic drainage and combined with surgical débridement of the chest in selected cases. The patients were examined with scores for dysphagia and fatigue.

\section{Materials and Methods}

Data from medical records of all 53 consecutive patients treated for esophageal perforation at Oslo University Hospital, Ullevål, from February 2007 to May 2015, were retrospectively registered in an Excel-created database. The etiology of the perforations were iatrogenic in 23 patients $(43 \%)$, foreign body in nine (15\%), postemetic in $17(32 \%)$ and miscellaneous in four (blunt trauma $(n=2)$, perforated esophageal ulcer, cancer) (7.5\%). The overall 30-day mortality was $9 \%$. In this paper we focused on the high risk group of 17 patients with spontaneous postemetic Boerhaave perforation. One patient, aged 89, with chronic obstructive lung disease and hemiparesis was excluded because she was too fragile for active treatment and died within one day. Another patient, aged 76, was excluded since the perforation initially was closed by suture. Accordingly, the 15 patients that received active treatment with initial stenting were further studied. The diagnosis was made by one or more of the 
following examinations; computed tomography (CT) scan with oral contrast of the thorax and upper abdomen, upper gastrointestinal endoscopy and chest x-ray. The self expanding metal stents (SEMS) used for sealing of the perforation were partially covered (Wallflex, Ultraflex) and fully covered (bleeding stent (SX-ELLA stent Danis), Niti-S, Polyflex). The preferred lengths varied from $9-15 \mathrm{~cm}$ and body diameter from $2.0-2.8 \mathrm{~cm}$. Treatment was initially based on antibiotics, sealing of the perforation by stenting, transthoracic drainage of the contaminated mediastinum and pleural cavities, usually with large diameter chest drains (usually 28-32 Fr). If the pleural cavity was very contaminated with gastric effluent and the patients general condition did not improve, the threshold was low for surgical débridement by thoracoscopy or thoracotomy.

Characteristics of the 15 patients receiving stent-based treatment are depicted in table 1. Alcohol and a heavy meal prior to emesis were involved in four of the patients. Such post-emetic perforations also occurred in two patients that had closure of a perforated duodenal ulcer and osteosynthesis of a malleolar fracture. One patient had a right colonic cancer that was resected after healing of the perforation. Ten patients still alive (67\%) at time of inquiry in August 2016, gave a written answer about dysphagia and fatique scores. The Ogilvie`s dysphagia score [4] from $0-4$ was used to determine ability to eat normal diet (score 0), swallow some solid foods (1) or only semi-solid foods (2) or liquids only (3) or unable to swallow (total dysphagia).

Total fatigue score consists of 11 items of graded questions with score $0-3$ per question, which is the sum of physical fatigue ( 7 items) and mental fatigue (4 items). This score has been validated in a Norwegian general population [5]. The respective scores for total, mental and physical fatigue are $0-33,0-21$ and $0-12$, and the higher score the more fatigue. The items of physical (1-7) and mental (8-11) fatigue were: 1) Do you have problems with tiredness? 2) Do you need to rest more? 3) Do you feel sleepy or drowsy? 4) Do you have problems with starting things? 5) Are you lacking in energy? 6) Do you have less strength in your muscles? 7) Do you feel weak? 8) Do you have difficulty concentrating? 9) Do you have problems thinking clearly? 10) Do you make slips of the tongue when speaking? 11) How is your memory? Student's t- test was used for comparison of fatigue scores between the patients and a Norwegian population based cohort [5] and p-values below 0.05 was considered statistically significant. 
The study was approved by the regional ethical committee (2012/1604/REK southeast (D) Norway).

\section{Results}

The length of the primary hospital stay was median 20 days (range $1-80$ days). Ten patients (67\%) were transferred to their local hospital. After a median observation time (August 2017) of 44 months (range $0-87$ months) 10 of the 15 patients (67\%) were alive. 30-day and overall in hospital mortality, including the patients' subsequent stay in their local hospital, was $13 \%(\mathrm{n}=2)$. Two patients, aged 50 and 56, died outside of hospital after 4 months from metastatic cancer and after 39 months from unknown cause, respectively. The third patient (aged 76) who initially was operated for a perforated duodenal ulcer, died in hospital after 34 months from a fatal peptic ulcer bleeding. The size of the perforation, documented in 13 out of 15 patients, was median $1.5 \mathrm{~cm}$ (average $1.6 \mathrm{~cm}$, range $0.5-3 \mathrm{~cm}$ ). The perforations were located within the $5 \mathrm{~cm}$ distal segment of the thoracic esophagus.

\section{Treatment}

The initial treatment of the patients is depicted in Table 2. All 15 patients received a SEMS in order to seal the perforation. The perforation was covered solely by the stent. Neither clips nor endoluminal suturing were performed in order to close the perforation or to anchor the stent in position. Moreover, drainage of the pleural cavities and débridement of contaminated left $(n=5)$ or right hemithorax $(n=3)$ was performed by thoracoscopy in five and by thoracotomy in three patients, respectively. Thirteen out of 15 patients received a nasoenteral tube for gastric decompression and enteral feeding. A patient, aged 73, with an overlooked perforation six days after suture of a perforated duodenal ulcer, was successfully treated with stenting and débridement through an open thoracotomy. A woman aged 56, was initially treated for a suspected pneumonia at another hospital by antibiotics, including percutaneous drainage of the pleural cavity. The overlooked esophageal perforation detected after 13 days, was treated by stenting and thoracotomy with débridement and decortication of the lung. This patient died from an aortic bleeding at day 14 caused by a chest drain injury confirmed by autopsy. 
The second patient with treatment related death was a woman aged 83 with dementia, who initially received drainage and a semicovered stent less than within 24 hours of the perforation. She was transferred to the local hospital and died from lung embolism after 15 days.

A second major treatment intervention more extensive than restenting, was necessary in four of the patients (27\%) because of severe complications. Patient 1, a man aged 39, had an operation for a malleolar fracture complicated with postoperative emesis and oesophageal perforation that was drained and stented within 24 hours. However, because of pleural contamination, reintervention with thoracotomy and débridement was necessary within after eight days. Patient 2, a woman aged 61, was initially stented for a perforation less than 48 hours after the debut of symptoms. Débridement through thoracosopy was also performed because of considerable pleural effusion and contamination. Despite initial débridement, at day 11 additional open access thoracic repeat operation was necessary. Patient 3, a man aged 69, despite initial thoracoscopic debridement after four days, a pleural empyema developed that was successfully removed through an open thoracotomy after 47 days. Patient 4 , a man aged 52, was stented within 24-48 hours and received a 32 Fr drain in the left pleural cavity. Upon of development of a pyothorax a thoracotomy with partial decortication of the lung was performed after 9 days. The patient was released from hospital after a stay of 70 days, and had a further uneventful course. Altogether 10 patients $(67 \%)$ had débridement, including twice in two patients.

\section{Complications}

Both partially covered stents (Wallstent $(n=1)$, Ultraflex $(n=1)$, unspecified $(n=2)$ and Wallflex $(n=4))$ and fully covered stents (Wallflex $(n=2)$, Polyflex $(n=2)$, bleeding stent ( $n=2)$, Niti-S (n=1) and unspecified stent $(n=1))$ were applied in order to seal the esophageal rupture. At initial stenting eight and seven patients received covered and partially covered stents, respectively, of whom one patient had both type of stents. Complications requiring restenting in 5 patients (33\%) were persistent leakage from a suboptimal stent position in three, stent migration in one and restenting in order to be able to remove the initial stent in one patient, respectively. Two stents were placed in four patients and 3 stents in one patient, respectively. The stents were finally removed after median 45 days (range $10-83$ ). Success rate for 
sealed leakage of the perforation by initial and restenting was $67 \%$ and $87 \%$, respectively. The perforations ultimately healed in all patients.

Based on the last upper endoscopy completed in our hospital, three out of the 15 patients $(20 \%)$ had detectable of esophageal stenosis. However, only two of the patients had dysphagia for some solid food (Ogilvie`s score 1). Patient 1, a man aged 69, had a stenosis with width $10 \mathrm{~mm}$ that was passable for the endoscope and dilation was not performed. Patient 2, a man aged 39, had a more prominent stricture not passable for the endoscope, that was dilated and subsequently treated with Argon plasma coagulation with improved effect. These two patients were adequately fed by oral nutrition. None of the patients had persistent leaks or fistulas. In recent years, there was a tendency to use more partially covered stents at initial stenting. Together with restenting, 11 patients $(73 \%)$ had complications (Table 3 ).

\section{Dysphagia and fatigue}

At time of inquiry (August 2016), nine out of 10 patients (90\%) responded to questions concerning dysphagia and fatigue scores postoperatively after median 25 months (range $8-45$ months). Their age was median 72 years (range $42-92$ ). The patients had a dysphagia score of median 0.5 (range 0 -2), of whom five scored 0 , three 1 and one 2.

Total fatigue score (mean $\pm \mathrm{SD}$ ) was $14.6 \pm 4.3$, while physical and mental scores were $10.5 \pm 3.9$ and $4.1 \pm 1.4$, respectively. For comparison, general Norwegian population based values in the age bracket above 60 years, based on 238 males [5], were $12.9 \pm 3.8(\mathrm{p}=0.05), 8.4 \pm 3.2(\mathrm{p}=0.19)$ and $4.5 \pm 1.2(\mathrm{p}=0.33)$.

\section{Discussion}

Spontaneous esophageal perforation, effort rupture (Boerhaave syndrome) is a lifethreatening condition with high morbidity and mortality [2], regardless of the treatment. We outline our experience with use of stenting and transthoracic drainage as primary approach, supplied by surgical débridement when there is an evolving mediastinal and pleural contamination.

The 30 days and in-hospital mortality of $13 \%$ in this patient material was similar to pooled mortality in Europe of $17.3 \%$ (95\% CI 14.0 - 20.8) based on 17 studies with iatrogenic and spontaneous perforations [3], initially treated with stenting or surgical repair. In a recent study with 16 patients treated for Boerhaave syndrome with sealing of 
the perforation by placement of SEMS, the results were similar with regard to the patients' age and their in-hospital mortality (13\%), but hospital stay was longer (median 35.5 days) [6]. However, early stenting after mean $22 \pm 33$ hours after perforation without mortality has been reported [7] in a relatively young group of 19 patients of mean age 48 years (range $26-67$ years). A concern in our study was the high proportion of patients with a delayed diagnosis and treatment (Table 1), underlined by the two patients with overlooked perforations who were treated for a perforated duodenal ulcer and a suspected pneumonia, respectively. Accordingly, the physician must be aware of this diagnosis and carry out relevant work-up based on esophagoscopy and an oral contrast CT scan, particularly in comorbid and elderly patients with emesis after heavy meals or in the immediate postoperative phase. A delayed diagnosis and treatment more than 24 hours after perforation increased mortality more than twofold [8]. In their study the time between onset of symptoms to accurate stent positioning was the only significant predictive factor for failed stent therapy. Consequently, a suboptimal stent position without sealing of the perforation must be revised as soon as possible.

In our study the ultimate leak occlusion rate and treatment success were $87 \%$, in line with an overall success rate of $88 \%$ reported in a meta-analysis of 371 patients stented for iatrogenic and spontaneous esophageal rupture [2]. In the surgical-based treatment [2] the data were similar with an overall success rate of $83 \%$ and an in-hospital mortality of $17 \%$ based on 368 patients. However, presumably the patients who underwent surgery probably were more severely afflicted compared with patients who underwent stent-based treatment.

We defined the treatment as successful if there were no signs of fistula and leakage on the last endoscopic examination, and that the patients were sufficiently fed by oral intake. Restenting was necessary in $\mathbf{3 3 \%}$ of the patients, mainly because of lack of sealing of the perforation. Moreover, four of the patients needed a second major intervention more extensive than restenting (débridement of the thorax cavity) in order to impove the overall outcome in these patients. This patient material dates back to the earliest days of stent-treatment of esophageal perforations in our department when altogether six different covered and partially covered stents were in use. Based on general clinical development and own experience partially covered stents have been preferred in later years, due to their significantly lower migration rate. Moreover, the 
uncovered flares in both ends often seal off leakage by ingrowth. However, at restenting covered stents were usually used. Infection induced edema of the aortic wall probably predisposed to the fatal aortic bleeding caused by the mediastinal drain.

Surgical decontamination of the chest was necessary in $67 \%$ of the patients. This was, at least to certain extent, caused by the fact that $60 \%$ received treatment beyond 24 hours after the time of perforation (Table 1). In the most severe cases of chest contamination, the procedure had to be performed through open access thoracotomy at initial treatment in three patients, including also as a repeat procedure in two patients, despite initial débridement by thoracoscopy. Therefore, in severe cases of chest contamination a thoracoscopic approach was insufficient for adequate clearance. In addition, two patients initially treated with transthoracic drainage within 24 hours and 24-48 hours, respectively, were also dependent on delayed débridement and decortication through thoracotomy. Consequently, in a setting of contaminated pleural effusion demonstrated by standard transthoracic drainage, the threshold should be low for débridement. If not, the patient is at high risk for development of a life-threatening pleural empyema and fistulisation to the airways and the thoracic wall. The sooner débridement is performed the more likely it can be done through a considerably less traumatic thoracoscopy than thoracotomy. Accordingly, our treatment approach was a combination of stenting and drainage and the mildest form of surgical approach based on preservation. In a recent study comparing stenting versus surgery for Boerhaave syndrome [9], operative intervention was necessary in as many as 11 of 13 patients initially stented $(85 \%)$ and the mortality was $15 \%$. This figure is comparable to our patient material, in which 10 out of the 15 patients $(67 \%)$ needed débridement of the thoracic cavity. Accordingly, stenting by no means precluded the need for surgical intervention, but successful sealing of the rupture by stenting may generally reduce the need for more radical surgery.

Surgery has been and still is the most common treatment for spontaneous rupture of the esophagus [1] but the use of initial stenting has gradually increased as shown in meta-analysis from 2000-2012 [3] and 2005 -2015 [2]. Moreover, there has been a tendency that the mortality after stent-based treatment was lower compared to surgical based treatment of iatrogenic and spontaneous perforations with pooled mortalities of $7.5 \%$ versus $17 \%[2]$. 
We think that stent-based and organ preserving treatment for spontaneous esophageal rupture is more optimal than resectional surgery, in order to obtain an optimal functional outcome, including food intake and digestive function.

In our series nine out of 10 patients reported after 25 months that they could feed themselves without supplemental nutrition. One patient could swallow only semi-solid food, whilst the remaining patients had no or only slight limitation of dysphagia.

This study showed a slight increase $(\mathrm{p}=0.05)$ in total fatigue, as could be expected, because of increased comorbidity, compared with a similar cohort of the general Norwegian population. The results must be interpreted with caution because of the low number of patients.

\section{Conclusion}

We conclude that spontaneous esophageal perforation initially can be treated safely by sealing of the perforation by insertion of a SEMS and additional chest drainage of the pleural effusion. Most patients require a combination of further stenting and débridement because of lack of sealing of the perforation and a contaminated mediastinum and pleural cavity. This should be done promptly, in order to reduce complications and mortality. The advantage of stenting is that the perforation healed in the majority of patients $(13 / 15 ; 87 \%)$ and that more invasive procedures requiring resection of the perforation and the gastric remnant was avoided (surgery). This because treatment based on organ preservation might result in a better functional outcome. Accordingly, more radical surgery, including resection and exclusion of the perforation should be used in severe cases, when organ conserving surgery is insufficient.

\section{Acknowledgements}

This research received no grant from any funding agency in the public, commercial or not-for-profit sectors.

\section{Disclosure statement}

The authors have no conflicts of interest or financial ties disclose. 


\section{References}

[1] Brinster CJ, Singhal S, Lawrence L, et al. Evolving options in the management of esophageal perforation. Ann Thorac Surg 2004; 77:1475-1483.

[2] Persson S, Rouvelas T, Lundell L. Outcomes following the main treatment options in patients with a leaking esophagus. Dis Esoph 2017;30:1-10.

[3] Biancari F, D'andrea V, Paone R, et al. Current treatment and outcome of esophageal perforations in adults: Systematic review and meta-analysis of 75 studies. World J Surg 2013;37:1051-1059.

[4] Ogilvie A, Dronfield MW, Ferguson R, Atkinson M. Palliative intubation of oesophagastric neoplasms at fibreoptic endoscopy. Gut 1982;23:1060-1067.

[5] Loge JH, Ekeberg O, Kaasa S. Fatigue in the general Norwegian population: normative data and associations. J Psychosom Res 1998;45:53-65.

[6] Glatz T, Marjanovic G, Kulemann B, et al. Management and outcome of esophageal stenting for spontaneous esophageal perforations. Dis Esoph 2017;30:1-6.

[7] Freeman RK, Van Woerkom JM, Vyverberg A, et al. Esophageal stent placement for the treatment of spontaneous esophageal perforations. Ann Thor Surg 2009;88:194-198.

[8] Persson S, Elbe P, Rouvelas I, et al. Predictors for failure of stent treatment for benign esophageal perforations - a single center 10-year experience. World J Gastroenterol 2014;20:10613-10619.

[9] Schweigert M, Beattie R, Solymosi N, et al. Endoscopic stent insertion versus primary operative management for spontaneous rupture of the esophagus (Boerhaave syndrome): an international study comparing the outcome. Am Surg 2013;79:634-640. 
Table 1. Characteristics of 15 patients with postemetic distal esophageal rupture.

\begin{tabular}{|c|c|}
\hline Men/women & +2 \\
\hline Median age (range) & $67.5(39-88)$ \\
\hline \multicolumn{2}{|l|}{ Comorbiditiy } \\
\hline - alcohol & 4 \\
\hline - nephritic syndrome & 1 \\
\hline - dementia & 1 \\
\hline Localisation (thoracic) & 15 \\
\hline \multicolumn{2}{|l|}{ Time to diagnosis and treatment } \\
\hline$<24 \mathrm{~h}$ & 6 \\
\hline $24-48 \mathrm{~h}$ & 4 \\
\hline$>72 \mathrm{~h}$ & 5 \\
\hline Perforation with pleural effusion & 15 \\
\hline - left chest & \\
\hline -right chest & \\
\hline -bilateral & \\
\hline
\end{tabular}


Table 2. Initial treatment of 15 patients with postemetic distal esophageal rupture.

Initial treatment

Stenting and débridement (thoracoscopy/thoracotomy) $5 / 3$

Stenting and drainage

6

Stenting

1

The patients received antibiotics. 
Table 3. Major complications in 11 out of 15 patients (73\%) treated for esophageal rupture.

Type of complication

Restenting in 7 because of

- leakage $(n=5)$

- stent migration $(\mathrm{n}=1)$

- removal of initial stent $(n=1)$

Pleural empyema $(n=4)$

Fatal aortic bleeding $(n=1)$

Lung arterial bleeding necessitating embolization $(\mathrm{n}=1)$

Lung embolism $(\mathrm{n}=1)$

Drain-induced lung laceration $(\mathrm{n}=1)$

Respiratory failure $(\mathrm{n}=1)$. 